\title{
Analysis of Treatment Response in Patients with Oral Lichen Planus
}

\author{
Hye-Min Ju', Kyung-Hee Kim², Hye-Mi Jeon ${ }^{3}$, Yong-Woo Ahn', Soo-Min Ok ${ }^{4}$, Sung-Hee Jeong ${ }^{4}$ \\ 'Department of Oral Medicine, Pusan National University Dental Hospital, Dental Research Institute, Yangsan, Korea \\ ${ }^{2}$ Department of Oral Medicine, Inje University Busan Paik Hospital, Busan, Korea \\ ${ }^{3}$ Dental Clinic Center, Pusan National University Hospital, Busan, Korea \\ ${ }^{4}$ Department of Oral Medicine, Pusan National University, School of Dentistry, Dental Research Institute, \\ Dental and Life Science Institute, Yangsan, Korea
}

Received May 28, 2021

Revised June 16, 2021

Accepted June 16, 2021

Correspondence to:

Sung-Hee Jeong

Department of Oral Medicine, Pusan

National University, School of Dentistry,

Dental Research Institute, Dental and Life

Science Institute, 49 Busandaehak-ro,

Mulgeum-eup, Yangsan 50612, Korea

Tel: +82-55-360-5242

Fax: +82-55-360-5238

E-mail:drcookie@pusan.ac.kr

https://orcid.org/0000-0002-6296-4775

This work was supported by a 2-Year

Research Grant of Pusan National University.
Purpose: To evaluate compliance by analyzing and comparing treatment duration, degree of improvement after treatment and treatment response of oral lichen planus (OLP) patients according to characteristics of them and the severity of the lesion.

Methods: According to treatment process, 132 subjects with OLP who first visited the Department of Oral Medicine at the Pusan National University Dental Hospital from January 2017 to December 2020were classified into three groups: Treatment completed (CT) group, Under treatment (UT) group, and Dropped out during follow-up (DT) group. The reticulation/keratosis, erythema, and ulceration (REU) scoring system was used to assess the severity of OLP. The degree of improvement after treatment was evaluated in CT group.

Results: There were 53 (40.15\%) CT, 27 (20.45\%) UT and 52 (39.39\%) DT. In CT group, according to initial REU score there was a statistical difference in the degree of improvement, but not in the length of time to complete treatment. There was no statistical difference between the days it took for patients to feel symptom relief, and the days of entire treatment among three groups. However, there was a positive correlation between the REU score of gingiva and duration of treatment in DT group. In the CT and DT groups, there was a correlation between the length of time taken to relieve symptoms and the duration of treatment.

Conclusions: The severity of the gingival lesion and the initial response to treatment have a large effect on the entire treatment period and prognosis, so it should be considered when explaining the disease prognosis and treatment period to patients, and the clinician needs to focus on initial symptom relief.

Key Words: Long-term care; Oral lichen planus; Patient compliance

\section{INTRODUCTION}

Oral mucosal diseases (OMD) include many types of diseases caused by various etiologies. Since oral mucosa show rapid recovery from damage, the treatment period is not long if the cause is removed [1]. In this instance, if the diagnosis is correct, the patient's discomfort can be quickly removed through appropriate treatment and medication provided and there is a high degree of patient cooperation and compliance. Conversely, OMD that require long-term treatment often develop into more serious OMD due to low patient compliance and poor treatment results [2].

Most of the OMD that require long-term treatment include autoimmune diseases such as oral lichen planus (OLP), mucosal pemphigoid, and mucosal pemphigus [3]. Of these, OLP is the most common disease affecting patient requiring long-term treatment for mucosal disease by the Department of Oral Medicine. It occurs mainly bilaterally and can occur

Copyright (C) 2021 Korean Academy of Orofacial Pain and Oral Medicine. All rights reserved.

(c) This is an open-access article distributed under the terms of the Creative Commons Attribution Non-Commercial License (http://creativecommons.org/licenses/by-nc/4.0/), which permits unrestricted non-commercial use, distribution, and reproduction in any medium, provided the original work is properly cited. 
anywhere in the oral cavity such as the buccal mucosa, tongue, and gingiva. Almost all lesions of OLP have a reticular form of white striae. Therefore, striae are also observed around erosive or ulcerative form. The clinical presentation of OLP occurs in a wide spectrum from patients showing only reticular form without any symptoms, to patients with multiple erythema and ulceration with severe symptoms $[4,5]$. Whilst there is a high correlation between the severity of OLP and the level of pain experienced by the patients, pain symptoms are not always present [6]. Many OLP patients are concerned about the possibility of malignancy in the lesion and the possibility of disease transmission. Although the actual rate of malignant transformation of OLP is only as low as $0 \%$ to $2 \%$, continuous follow-up is very important as there is still a risk of conversion [7].

Steroids are the main option used in the treatment of OLP and are available in various dosage forms such as gargles, ointments, lesional injections, and pills [8]. Topical agents are most commonly used to treat, however, they present a challenge for patient compliance as the treatment require patients to keep their mouth closed post application. In clinically, this requires a high degree of patient cooperation and diligence, especially when socializing. Therefore, differences in compliance to treatment in OLP patients lead to differences in treatment outcomes [2].

This study aims to evaluate compliance by analyzing and comparing treatment duration, the degree of improvement after treatment, and treatment response according to the characteristics of the patient's initial visit and the severity of the lesion.

\section{MATERIALS AND METHODS}

\section{Subjective}

Patients with OLP were recruited from those who first visited the Department of Oral Medicine at the Pusan National University Dental Hospital from January 2017 to December 2020. Patients who visited more than 3 times were included. The following set of exclusion criteria was then applied to the potential cohort of 226 patients: subjects with other oral lesions, those who are taking corticosteroids or immunosuppressive medications at that time due to OLP or other systemic diseases, or had a record of taking them within 6 months, patients who could not confirm treatment results due to no clinical photo, and patients with dysplasia as a result of biopsy. As a result, a total of 132 patients were selected for the study (Fig. 1). The patients were grouped according to the following characteristics: sex, age, systemic disease of the patients, and the severity of OLP, which was analyzed using clinical photo through the reticulation/keratosis, erythema, and ulceration (REU) scoring system. The medicines applied at each visit were investigated, and the total treatment period of the patients as well as the time period until the subjective symptoms of the patient resolved were investigated. This study was approved by the Institutional Review Board of Pusan National University

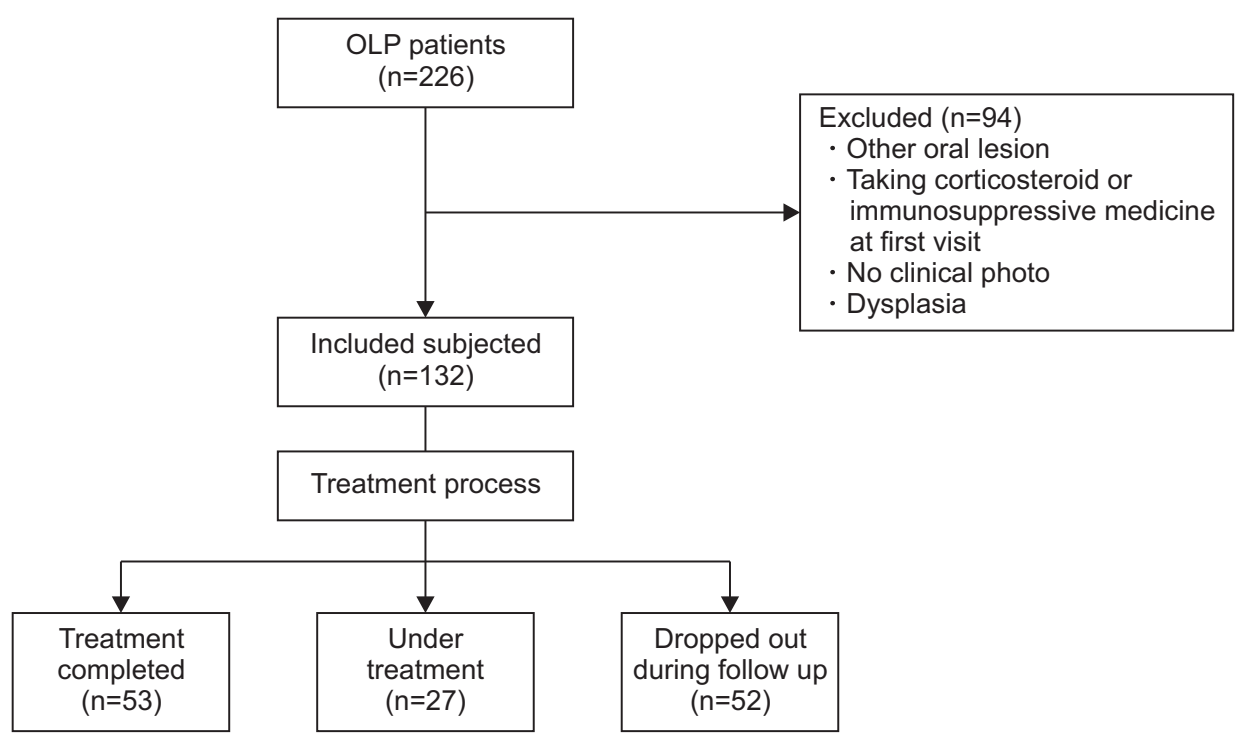

Fig. 1. Subject flow chart. OLP, oral lichen planus. 
Dental Hospital (IRB No. PNUDH-2021-019). Written informed consent was obtained from all patients at the initial visit.

\section{Reticulation/Keratosis, Erythema, and Ulceration Scoring System and Grouping}

Based on the REU criteria, the oral cavity was divided into 10 sites (upper/lower labial mucosa, right buccal mucosa, left buccal mucosa, dorsal tongue, ventral tongue, floor of the mouth, hard palate mucosa, soft palate/tonsillar pillars, maxillary gingiva, and mandibular gingiva), and 0 or 1 point was given to each part for the existence or nonexistence of a reticular/hyperkeratotic form. In addition, if there was no erythema or ulcer, 0 points were given. One point was given for lesions smaller than $1 \mathrm{~cm}^{2}, 2$ points for 1 to $3-\mathrm{cm}^{2}$ lesions, and 3 points for lesions greater than $3 \mathrm{~cm}^{2}$. The final score was calculated by summing the total scores of reticular, erythema, and ulcer for each clinical sign, with weights of 1, 1.5, and 2 given to them, respectively [9].

Based on the REU score, OLP severity was divided into three groups: Mild (0 to 5), Moderate (5.5 to 15), and Severe (15.5 or more). According to the REU scoring system, 5 points or less can be scored only when there is an erythema or ulcer smaller than $1 \mathrm{~cm}^{2}$ with a reticular form, hence, this group was called mild. The severe group with a score of 15.5 points or more must have at least one reticular form site with at least two erythema with a size of $3 \mathrm{~cm}^{2}$ or more and an ulcer with a size of $3 \mathrm{~cm}^{2}$ or more in at least one site. Treatment response analysis was evaluated by dividing into three groups according to the score.

According to the treatment process, 132 subjects were classified into three groups: Treatment completed (CT), Under treatment (UT), and Dropped out during follow-up (DT) (Fig. 1). CT in this study refers to a case where erosive and ulcer lesions do not exist, stating that the patient is using only the steroid gargle solution and is only required to have a follow-up assessment every 3 months or more and that the patient has no discomfort in their daily life.

\section{Criteria for Evaluating the Degree of Improvement in Treatment}

Various types of steroid agents such as gargles, ointments, lesional injections (triamcinolone injection $40 \mathrm{mg} / \mathrm{mL}$ ) and pills (prednisolone) have been used for the treatment of OLP patients. As for topical ointments, clobetasol propionate 0.05\% and fluocinonide 0.05\% were used as high potency, dexamethasone sodium phosphate $0.1 \%$ as low potency [10].

$\mathrm{T}_{0}$ refers to the REU score at the initial visit and $\mathrm{T}_{\mathrm{F}}$ is the score after the final treatment. The efficacy index of clinical REU improvement was calculated using the formula $\mathrm{T}(\%)=\left[\left(\mathrm{T}_{0}-\mathrm{T}_{\mathrm{F}}\right) \div \mathrm{T}_{0}\right] \times 100$. The efficacy index was classified as follows to determine the level of improvement: (1) Complete healing: efficacy index of 100\%; (2) Marked improvement: 70\%<efficacy index<100\%; (3) Moderate improvement: 30\%<efficacy index<70\%; (4) No improvement: efficacy in$\operatorname{dex}<30 \%[11]$.

\section{Statistical Analysis}

All statistical analyses were performed using IBM Statistical Package for the Social Sciences (SPSS) version 22.0 (IBM Corp., Armonk, NY, USA). A chi-squared test was used to determine distribution differences in sex, ointment used, and diabetes mellitus (DM) status between groups. After $T_{F}$ in the treated group, a chi-squared test was also used to determine whether there was a difference in the degree of improvement after treatment between the three groups. If the expected frequency of the cell was lower than 5, Fisher's exact test was used. Kruskall-Wallis was used to determine the difference in age, the period until treatment completion, the REU score at the initial visit, and the REU score at the time of treatment completion between groups. The ANOVA test was used to determine the difference in REU scores, time taken to symptom relief, and the time taken until the final visit between CT, UT and DT groups at the time of investigation. The Spearman rho correlation was used to investigate the correlation between treatment duration and REU score at the initial visit. $\mathrm{p}<0.05$ was considered to be statistically significant.

\section{RESULTS}

Using the REU score at the initial visit, the 132 eligible OLP patients were grouped as follow: 58 in the mild group (43.94\%), 58 in the moderate group (43.94\%), and 16 in the severe group (12.12\%). There were no differences in the age, sex, and proportions of diabetic patient between groups 
(Table 1). There was no statistical difference between groups in systemic diseases (data not shown). There were statistical differences in REU scores for sites and the total mean between the groups divided by mild, moderate, and severe according to the REU score $(\mathrm{p}<0.001$; Table 1$)$.

Of the 132 patients, 53 (40.15\%) CT, 27 (20.45\%) of the remaining 79 were UT, and 52 (39.39\%) were DT. Of the 53 CT, 24 patients $(45.28 \%)$ were in the mild, 25 (47.17\%) were in the moderate, and 4 (7.55\%) were in the severe group according to the REU score at the initial visit. There was no statistically significant difference in the efficacy of the ointment used in the patients and whether or not the patients ware diabetic. In addition, there was no statistical difference in the time taken to relieve symptoms by group, and there was no statistical difference in the time taken to complete treatment (Table 2). However, there was a statistical difference in the degree of improvement after treatment at completion ( $\mathrm{p}<0.01$; Table 2 ). The mild group showed a high percentage of complete healing at $41.7 \%$, but no improvement was also 20.8\% compared to the moderate and severe groups. In the moderate group, the degree of complete healing was $16.0 \%$, which was lower than that of the mild group, but the patient with no improvement did not exist, and complete healing patients did not exist in the severe group (Table 2).

When comparing the characteristics between the CT, UT

Table 1. Characteristics of the patients at $T_{0}$

\begin{tabular}{|c|c|c|c|c|c|}
\hline Characteristic & Total $(n=132)$ & Mild $(n=58)$ & Moderate $(n=58)$ & Severe $(n=16)$ & p-value \\
\hline Age (y) & $59.63 \pm 10.63$ & $58.18 \pm 11.8$ & $60.57 \pm 9.25$ & $61.50 \pm 10.66$ & 0.388 \\
\hline Sex & & & & & 0.691 \\
\hline Female & $97(73.5)$ & $41(70.7)$ & $43(74.1)$ & $13(81.3)$ & \\
\hline Male & $35(26.5)$ & $17(29.3)$ & $15(25.9)$ & $3(18.8)$ & \\
\hline Diabetes mellitus & & & & & 0.265 \\
\hline Diagnosed & $24(18.2)$ & $7(12.1)$ & $13(22.4)$ & $4(25.0)$ & \\
\hline Not diagnosed & $108(81.8)$ & $51(87.9)$ & $45(77.6)$ & $12(75.0)$ & \\
\hline Labial mucosa & $0.38 \pm 0.84$ & $0.22 \pm 0.63$ & $0.32 \pm 0.60$ & $1.16 \pm 1.60$ & $0.000 * * *$ \\
\hline Buccal mucosa & $4.18 \pm 4.18$ & $1.57 \pm 1.27$ & $4.81 \pm 3.12$ & $11.34 \pm 5.18$ & $0.000 * * *$ \\
\hline Tongue & $1.08 \pm 1.87$ & $0.28 \pm 0.56$ & $1.42 \pm 2.07$ & $2.78 \pm 2.67$ & $0.000 * * *$ \\
\hline Palate & $0.16 \pm 1.01$ & $0.00 \pm 0.00$ & $0.09 \pm 0.40$ & $1.00 \pm 2.74$ & $0.001 * *$ \\
\hline Gingiva & $2.62 \pm 3.66$ & $0.91 \pm 1.40$ & $2.87 \pm 2.72$ & $7.91 \pm 6.32$ & $0.000 * * *$ \\
\hline REU score & $8.26 \pm 7.08$ & $2.97 \pm 1.35$ & $9.42 \pm 2.81$ & $23.19 \pm 7.05$ & $0.000 * * *$ \\
\hline
\end{tabular}

$\mathrm{REU}$, reticulation/keratosis, erythema, and ulceration.

Values are presented as mean \pm standard deviation or number (\%). p-values were obtained by one-way Kruskal - Wallis and chi-squared test. $* * p<0.01 ; * * * p<0.001$.

Table 2. Characteristics of the treatment completed group patients $(n=53)$ with stable sign and symptom after treatment

\begin{tabular}{|c|c|c|c|c|}
\hline Characteristic & Mild $(n=24)$ & Moderate $(n=25)$ & Severe $(n=4)$ & $p$-value \\
\hline \multicolumn{5}{|l|}{ Topical steroid } \\
\hline Low-potency & $9(37.5)$ & $6(24.0)$ & $3(75.0)$ & 0.120 \\
\hline High-potency & $15(62.5)$ & $19(76.0)$ & $1(25.0)$ & \\
\hline \multicolumn{5}{|l|}{ Diabetes mellitus } \\
\hline Diagnosed & $2(8.3)$ & $8(32.0)$ & $0(0.0)$ & 0.064 \\
\hline Not diagnosed & $22(91.7)$ & $17(68.0)$ & $4(100.0)$ & \\
\hline Time taken for symptom relief (day) & $40.04 \pm 24.58$ & $51.04 \pm 48.88$ & $45.25 \pm 28.12$ & 0.618 \\
\hline Time taken for complete treatment (day) & $354.52 \pm 283.75$ & $219.79 \pm 142.03$ & $141.00 \pm 47.40$ & 0.162 \\
\hline \multicolumn{5}{|l|}{ The degree of improvement in treatment } \\
\hline Complete healing & $10(41.7)$ & $4(16.0)$ & $0(0.0)$ & $0.007^{* *}$ \\
\hline Marked improvement & $3(12.5)$ & $13(52.0)$ & $3(75.0)$ & \\
\hline Moderate improvement & $6(25.0)$ & $8(32.0)$ & $1(25.0)$ & \\
\hline No improvement & $5(20.8)$ & $0(0.0)$ & $0(0.0)$ & \\
\hline
\end{tabular}

Values are presented as number (\%) or mean \pm standard deviation. $\mathrm{p}$-values were obtained by one-way Kruskal - Wallis and chi-squared test. $* * p<0.01$. 
and DT groups, there was no statistical difference between groups in REU scores for other intraoral sites and the total score at the initial visit, but the REU score for tongue in UT group was higher on average $(\mathrm{p}<0.01$; Table 3$)$. There was no statistical difference between the days it took for patients to feel symptom relief and the final treatment period between the three groups.

When examining the correlation between the total treatment duration of patients, REU score and duration of symptom relief, there was a negative correlation between REU scores of labial mucosa and gingiva and duration of treatment in the CT group, and the UT group had no correlation between duration and score. There was a positive correlation between the REU score of labial mucosa and the duration of treatment in the DT group, and there was also a correlation with the REU score of gingiva. In the CT and DT groups, there was a correlation between the time taken to relieve symptoms and the duration of treatment (Table 4).

\section{DISCUSSION}

Patients visited the hospital with various degrees of disease severity, and because one or more treatment methods were applied based on the severity of the disease, the effectiveness of a specific treatment could not be compared so this could not be used as an evaluation item. Therefore, characteristics were compared by classifying them according to the initial severity, not according to the treatment type, and the characteristics of patients according to the difference in the treatment process at the time of the final investigation were compared. This study was conducted over four-year period from 2017 to 2020, and at the time of the final investigation, patients who were still undergoing treatment or did not visit the hospital during treatment might have the possibility of completing treatment or revisit in the future. In other words, these patients were more likely to return for follow-up but could not be included in CT group, thus limitations that may have had an effect on

Table 3. Differences in characteristics of patients according to treatment progress

\begin{tabular}{lccccc}
\multicolumn{1}{c}{ Characteristic } & $\begin{array}{c}\text { CT group } \\
(\mathrm{n}=53)\end{array}$ & $\begin{array}{c}\text { UT group } \\
(\mathrm{n}=27)\end{array}$ & $\begin{array}{c}\text { DT group } \\
(\mathrm{n}=52)\end{array}$ & $\begin{array}{c}\text { Post-Hoc } \\
\text { result }\end{array}$ \\
\hline Labial mucosa & $0.43 \pm 0.88$ & $0.28 \pm 0.59$ & $0.37 \pm 0.92$ & 0.735 & 0.967 \\
Buccal mucosa & $4.08 \pm 3.85$ & $4.17 \pm 3.67$ & $4.29 \pm 4.78$ & $0.004^{\star *}$ & $2>1,3$ (Scheffe) \\
Tongue & $0.81 \pm 1.49$ & $2.15 \pm 2.74$ & $0.81 \pm 1.47$ & 0.653 & 0.609 \\
Palate & $0.30 \pm 1.69$ & $0.09 \pm 0.34$ & $0.13 \pm 0.65$ & 0.453 \\
Gingiva & $2.27 \pm 3.80$ & $3.11 \pm 3.22$ & $2.72 \pm 3.75$ & 0.370 \\
Total REU score & $7.59 \pm 6.58$ & $9.70 \pm 6.79$ & $8.18 \pm 7.71$ & 0.072 \\
Time taken for symptom relief (day) & $45.73 \pm 38.21$ & $62.82 \pm 38.24$ & $72.28 \pm 142.98$ & \\
Length of time from first to last visit (day) & $293.34 \pm 257.44$ & $444.85 \pm 372.39$ & $296.21 \pm 299.66$ &
\end{tabular}

$\mathrm{CT}$, Treatment completed; UT, Under treatment; DT, Drop out during follow-up; REU, reticulation/keratosis, erythema, and ulceration. Values are presented as mean \pm standard deviation. $p$-values were obtained by ANOVA test.

$* * \mathrm{p}<0.01$.

Table 4. Correlations between REU score and treatment period

\begin{tabular}{|c|c|c|c|c|c|c|c|c|c|}
\hline Treatment period & Group & Value & $\begin{array}{l}\text { Labial } \\
\text { mucosa }\end{array}$ & $\begin{array}{c}\text { Buccal } \\
\text { mucosa }\end{array}$ & Tongue & Palate & Gingiva & $\begin{array}{l}\text { REU } \\
\text { score }\end{array}$ & $\begin{array}{l}\text { Number of days } \\
\text { until symptom } \\
\text { relief }\end{array}$ \\
\hline \multirow{6}{*}{ From first visit to last visit } & $C T(n=53)$ & $r$ & -0.331 & -0.025 & -0.071 & -0.135 & -0.115 & -0.116 & 0.077 \\
\hline & & $\mathrm{p}$ & $0.015^{\star}$ & 0.857 & 0.611 & 0.335 & $0.025^{*}$ & 0.120 & $0.006 * *$ \\
\hline & UT $(n=27)$ & $r$ & 0.101 & 0.009 & -0.047 & 0.028 & 0.125 & -0.066 & 0.315 \\
\hline & & $\mathrm{p}$ & 0.615 & 0.966 & 0.817 & 0.889 & 0.535 & 0.744 & 0.154 \\
\hline & DT $(n=52)$ & $r$ & 0.460 & 0.028 & 0.011 & -0.196 & 0.305 & 0.311 & 0.342 \\
\hline & & $\mathrm{p}$ & $0.001 * *$ & 0.846 & 0.938 & 0.164 & $0.028^{*}$ & $0.025^{*}$ & $0.020 *$ \\
\hline
\end{tabular}

REU, reticulation/keratosis, erythema, and ulceration; CT, Treatment completed; UT, Under treatment; DT, Drop out during follow-up. p-values were obtained by Spearman rho.

$*_{p}<0.05 ;{ }^{*} p<0.01$. 
the results. Nevertheless, there was no statistical difference in the duration for the final visit at the time of the previous four years of investigation, so it is clinically meaningful to compare the patient groups according to the difference in treatment process at the time of the investigation (Table 3). The characteristics of the patients at the initial visit showed the same results as previous studies in age and sex [12]. In addition, DM is considered a risk factor for OLP development, and It was reported that OLP patients have a significant relationship with non-insulin-dependent DM compared to normal population $[13,14]$. In previous study that analyzed the relationship between DM and OLP prevalence, the prevalence of OLP in DM patients was $1.6 \%$ to $37.7 \%$, and a 2.432 -fold relative risk was reported, and the prevalence of DM in OLP patients was $0.5 \%$ to $6.1 \%$, and the relative risk was 1.4 times [15]. In this study, there were approximately $18.2 \%$ of OLP patients with DM, which was a higher proportion than that of the general population of South Korea, which has a prevalence of diabetes in men of $2.26 \%$ and in women of 2.03\% [16]. However, other studies reported that DM had no direct relationship with the cause of OLP, and the relationship between DM and OLP is still debated [17]. In this study, the ratio was higher than that of normal people, but there was no statistical difference between the groups according to OLP severity and the ratio of diabetes patients and the same result was shown in the CT group (Table 1). A variety of other systemic diseases were also investigated, including hypertension, hyperlipidemia, cardiac disease, asthma, thyroid disease, hepatitis B and 52 (39.39\%) of the 132 patients had one or more systemic disorders. Among them, there was one patient with hepatitis $\mathrm{C}$ virus infection, which was highly related to OLP. However, there was no statistical relationship between the type of systemic disease and the groups according to the severity of OLP (data not shown) [12].

OLP is a chronic disease that is not cured and requires continuous follow-up. However, patients demonstrating a high level of treatment compliance usually experience no sign and symptom of discomfort, and can easily manage the disease with only intermittent use of topical medications. When these patients were referred to as the CT group, the Moderate and Severe group showed a degree of improvement above the moderate level. However, the complete healing rate was low or nonexistent, whereas the Mild group showed either complete healing or no improvement in some patients (Table 2). This was largely influenced by a reticular form, which is known to require no treatment if there are no symptoms [18]. However, in practice, there are some patients with reticular form who show improvement with corticosteroid treatment, and others who do not respond at all $[4,19]$. The reason that the patient's subjective symptom relief time showed similar results according to OLP severity is thought to be due to differences in patient expectations for treatment effect. Patients with mild conditions are more likely to expect complete healing, and patients with moderate and severe conditions are considered to have improved by showing a certain level of improvement in treatment compared to before treatment. In addition, there was no statistical difference in the actual mitigation period of sign and symptom, which is thought to be because the drug was selected and applied according to the severity of the patient's physician and patient preference. The Moderate and Severe groups used a combination of strong steroids such as systemic corticosteroid and injection from the first visit, and the mild group used gargles and topical agents.

Symptoms of OLP according to the subjectiveness and perception of the patient, resulting in some patients do not recognizing lesion, whilst others complain of severe pain [4]. The UT group in this study showed a higher REU score of tongue compared to the other two groups (Table 3). The tongue first contacts food and is a movable structure in the oral cavity that has a major effect on the patient's symptoms. Previous studies that investigated predictors of symptomatic OLP also found that the probability of symptoms increased by 2.3 times when the lesion was located on the tongue [20]. OLP is most common in buccal mucosa, but patients with gingival lesions are easily stimulated by brushing or eating food, and it is difficult to control plaque, which leads to a long treatment period (Table 4). As in this study, if symptoms are not eased well or the treatment period itself is prolonged, patients' adherence to treatment decreases, and they are likely to drop out during follow-up [21]. However, it is not easy to explain long-term care to patients who want a quick and complete cure of the disease and make them visit the hospital regularly. The current goal 
of OLP therapy is to treat mucosal erythema and ulceration, relieve symptoms, and reduce the risk of oral cancer [22]. OLP should also be aimed at maintaining improved conditions, such as diabetes and high blood pressure, rather than at the concept of complete cure, so that all patients should be educated on the characteristics of the disease at the first visit and the importance of periodic follow-ups should be emphasized to patients.

Patients with good treatment progress required an average of one year for the total treatment period regardless of the severity of the lesion and commonly experienced symptom relief at the beginning of treatment. In particular, symptom relief of gingival lesions had an important effect on the treatment period. Most of the patients in the CT group took a short time to relieve symptoms even when the initial gingival lesion was severe. In the DT group, it took a long time for gingival lesions symptoms to be relieved during the treatment period. In actual clinical practice, lesions of the buccal mucosa are the most common, but the response to treatment does not significantly affect the patient's compliance, whereas the response of the gingival lesions affects the patient's compliance. Therefore, since the severity of the gingival lesion and the initial response to treatment have a large effect on the treatment period and prognosis, so it should be considered when explaining the disease prognosis and treatment period to patients, and the clinician needs to focus on initial symptom relief.

\section{CONFLICT OF INTEREST}

No potential conflict of interest relevant to this article was reported.

\section{ORCID}

Hye-Min Ju

https://orcid.org/0000-0002-9252-6717

Kyung-Hee Kim

https://orcid.org/0000-0003-2922-6452

Hye-Mi Jeon

https://orcid.org/0000-0003-0007-5662

Yong-Woo Ahn

https://orcid.org/0000-0002-2197-0394

\author{
Soo-Min Ok \\ https://orcid.org/0000-0003-1776-371X \\ Sung-Hee Jeong \\ https://orcid.org/0000-0002-6296-4775
}

\section{REFERENCES}

1. Stoopler ET, Sollecito TP. Oral mucosal diseases: evaluation and management. Med Clin North Am 2014;98:1323-1352.

2. Gonzalez-Moles MA, Bravo M, Gonzalez-Ruiz L, Ramos P, GilMontoya JA. Outcomes of oral lichen planus and oral lichenoid lesions treated with topical corticosteroid. Oral Dis 2018;24:573579.

3. Sciubba JJ. Autoimmune oral mucosal diseases: clinical, etiologic, diagnostic, and treatment considerations. Dent Clin North Am 2011;55:89-103.

4. Chainani-Wu N, Silverman S Jr, Lozada-Nur F, Mayer P, Watson JJ. Oral lichen planus: patient profile, disease progression and treatment responses. J Am Dent Assoc 2001;132:901-909.

5. Sugerman PB, Savage NW. Oral lichen planus: causes, diagnosis and management. Aust Dent J 2002;47:290-297.

6. Park HK, Hurwitz S, Woo SB. Oral lichen planus: REU scoring system correlates with pain. Oral Surg Oral Med Oral Pathol Oral Radiol 2012;114:75-82.

7. Shirasuna K. Oral lichen planus: malignant potential and diagnosis. Oral Sci Int 2014;11:1-7.

8. Lodi G, Manfredi M, Mercadante V, Murphy R, Carrozzo M. Interventions for treating oral lichen planus: corticosteroid therapies. Cochrane Database Syst Rev 2020;2:CD001168.

9. Piboonniyom SO, Treister N, Pitiphat W, Woo SB. Scoring system for monitoring oral lichenoid lesions: a preliminary study. Oral Surg Oral Med Oral Pathol Oral Radiol Endod 2005;99:696-703.

10. Kwatra G, Mukhopadhyay S. Topical corticosteroids: pharmacology. In: Lahiri K, ed. A treatise on topical corticosteroids in dermatology. Singapore: Springer; 2018. pp. 11-22.

11. Liu J, Zeng X, Chen Q, et al. An evaluation on the efficacy and safety of amlexanox oral adhesive tablets in the treatment of recurrent minor aphthous ulceration in a Chinese cohort: a randomized, double-blind, vehicle-controlled, unparallel multicenter clinical trial. Oral Surg Oral Med Oral Pathol Oral Radiol Endod 2006;102:475-481.

12. Lavanya N, Jayanthi P, Rao UK, Ranganathan K. Oral lichen planus: an update on pathogenesis and treatment. J Oral Maxillofac Pathol 2011;15:127-132.

13. Ahmed I, Nasreen S, Jehangir U, Wahid Z. Frequency of oral lichen planus in patients with noninsulin dependent diabetes mellitus. J Pak Assoc Dermatol 2012;22:30-34.

14. Romero MA, Seoane J, Varela-Centelles P, Diz-Dios P, GarciaPola MJ. Prevalence of diabetes mellitus amongst oral lichen planus patients. Clinical and pathological characteristics. Med Oral 2002;7:121-129.

15. Otero Rey EM, Yáñez-Busto A, Rosa Henriques IF, López-López J, Blanco-Carrión A. Lichen planus and diabetes mellitus: system- 
atic review and meta-analysis. Oral Dis 2019;25:1253-1264.

16. Kim SA, Park WS, Ohrr HC, et al. Prevalence and management status of diabetes mellitus in Korea. Korean J Med 2005;68:1017.

17. Bagewadi A, Bhoweer AK. Oral lichen planus and its association with diabetes mellitus and hypertension. J Indian Acad Oral Med Radiol 2011;23:300-303.

18. Edwards PC, Kelsch R. Oral lichen planus: clinical presentation and management. J Can Dent Assoc 2002;68:494-499.

19. Fernández-González F, Vázquez-Álvarez R, Reboiras-López D, Gándara-Vila P, García-García A, Gándara-Rey JM. Histopathological findings in oral lichen planus and their correlation with the clinical manifestations. Med Oral Patol Oral Cir Bucal 2011;16:e641-e646.

20. Osipoff A, Carpenter MD, Noll JL, Valdez JA, Gormsen M, Brennan MT. Predictors of symptomatic oral lichen planus. Oral Surg Oral Med Oral Pathol Oral Radiol 2020;129:468-477.

21. Graber AL, Davidson P, Brown AW, McRae JR, Woolridge K. Dropout and relapse during diabetes care. Diabetes Care 1992;15:1477-1483.

22. Boorghani M, Gholizadeh N, Taghavi Zenouz A, Vatankhah M, Mehdipour M. Oral lichen planus: clinical features, etiology, treatment and management; a review of literature. J Dent Res Dent Clin Dent Prospects 2010;4:3-9. 